\title{
ON THE SOLUTION OF A NONLINEAR PROBLEM IN CELL MEMBRANE THEORY
}

\author{
A. M. ARTHURS and J. CLEGG ${ }^{1}$
}

(Received 21 September 1992; revised 2 July 1993)

\begin{abstract}
Methods for integral equations are used to derive upper and lower pointwise bounds for the solution of a nonlinear boundary value problem arising in the steady-state finite cable model of cell membranes. Test calculations are performed to illustrate the results and the accuracy achieved is significantly better than that obtained previously by other methods.
\end{abstract}

\section{Introduction}

Boundary value problems described by equations of the form

$$
\frac{d u}{d x}=-i, \quad-\frac{d i}{d x}=m(u), \quad 0<x<1,
$$

with

$$
u(0)=u_{0}, \quad i(1)=0,
$$

arise in the steady-state finite cable model of nonlinear cell membranes [3]. Here $x$ measures the distance down a finite one-dimensional cable, $u_{0}$ is a prescribed positive number, current is injected at the end $x=0$ and the end $x=1$ is terminated in an open circuit, $u(x)$ is the transmembrane potential and $i(x)$ is the axial or longitudinal current down the core of the cable. The membrane current per unit length $m(u)$ can be any linear or nonlinear function of $u$ which crosses the $m(u)=0$ axis only once with a positive slope. The values of $u(1)$ and $i(0)$ are not known and must be determined as part of the solution.

Several approaches to solving problems like this with nonlinear $m(u)$ have been described [1, 2, 4]. The numerical approach of Kootsey [4] combined a shooting method with an automatic algorithm for iteration, and involved initial estimates of

'Department of Mathematics, University of York, England

(C) Australian Mathematical Society, 1994, Serial-fee code 0334-2700/94 
the unknown input current. The variational approach of Anderson and Arthurs [1] employed extremum principles as the basis of a practical optimisation procedure. Simple analytical bounding curves for the solution have also been obtained [2] and these show that the solution is known in the cases considered to within a few per cent. Such accuracy may suffice for certain applications, but to improve on this accuracy is of theoretical and practical interest and it is this aspect of the problem that we shall consider here. Of the methods already used the one producing bounding curves provides an immediate and direct measure of the error at each stage. However in the quest to reduce the error this method becomes progressively more cumbersome and slow, since it employs polygonal approximations to $m(u)$. For that reason we have decided to investigate a quite different approach using an integral equation formulation, some simple bounding results, and an approximation scheme based on an inclusion procedure. This approach proves to be straightforward to implement and in the test cases considered leads to very significant improvements in accuracy.

\section{Boundary value problem}

In terms of the function $u$ the boundary value problem of (1.1) and (1.2) is

$$
\frac{d^{2} u}{d x^{2}}=m(u) \quad(0<x<1),
$$

with

$$
u(0)=u_{0}, \quad u^{\prime}(1)=0 . \quad\left(u_{0}>0\right) .
$$

For the purposes of this paper we shall consider the case of a third order polynomial membrane current-voltage relation given by

$$
m(u)=u+u^{3}
$$

This relation is used to represent steady-state outward-going rectification observed in nerve membranes [3]. With $m(u)$ of the form (2.3) it follows immediately, by a simple convexity argument using (2.1) and the fact that $u(0)=u_{0}>0$, that the solution $u$ satisfies

$$
0 \leq u \leq u_{0} \quad(0 \leq x \leq 1) .
$$

We shall now rewrite the problem in the form

$$
A u=h(u) \quad(0<x<1),
$$

with

$$
u(0)=u_{0}, \quad u^{\prime}(1)=0 \quad\left(u_{0}>0\right),
$$


in which

$$
\begin{aligned}
A u & =-u^{\prime \prime}+\sigma^{2} u, \\
h(u) & =\left(\sigma^{2}-1\right) u-u^{3},
\end{aligned}
$$

where $\sigma$ denotes a free real parameter at this stage. Next, in order to work with homogeneous boundary conditions, we split the function $u$ by writing

$$
u=\varphi(x)+\xi(x) .
$$

Then (2.5) and (2.6) give

$$
A \varphi+A \xi=h(\varphi+\xi), \quad(0<x<1),
$$

with

$$
\varphi(0)+\xi(0)=u_{0}, \quad \varphi^{\prime}(1)+\xi^{\prime}(1)=0 .
$$

We now choose $\xi$ to satisfy

$$
\begin{aligned}
A \xi & =0, \quad(0<x<1), \\
\xi(0) & =u_{0}, \quad \xi^{\prime}(1)=0,
\end{aligned}
$$

which leads to

$$
\xi(x)=u_{0} \cosh \sigma(1-x) / \cosh \sigma .
$$

It then follows that the function $\varphi$ satisfies

$$
A \varphi=f(\varphi) \quad(0<x<1),
$$

with

$$
\varphi(0)=0, \quad \varphi^{\prime}(1)=0,
$$

in which

$$
f(\varphi)=h(\varphi+\xi)=\left(\sigma^{2}-1\right)(\varphi+\xi)-(\varphi+\xi)^{3},
$$

where $\xi$ is the function given by (2.14). The problem in (2.5) and (2.6) has thus been transformed to that of finding the solution $\varphi$ of (2.15) and (2.16). We shall regard the solution $\varphi$ as belonging to the real Hilbert space $\mathcal{L}_{2}(0,1)$ with inner product

$$
\langle\psi, \zeta\rangle=\int_{0}^{1} \psi(t) \zeta(t) d t
$$

and associated norm

$$
\|\varphi\|=\langle\varphi, \varphi\rangle^{\frac{1}{2}}
$$


In addition we know from (2.4) that the solution function $\varphi(x)$ belongs to the subset of $\mathcal{L}_{2}(0,1)$ defined by

$$
\mathcal{S}=\left\{\varphi_{i}(x): 0 \leq \varphi_{i}+\xi \leq u_{0}, \quad 0 \leq x \leq 1\right\} .
$$

Relative to this space the operator

$$
A=-\frac{d^{2}}{d x^{2}}+\sigma^{2} I
$$

in (2.15), with (2.16), is self-adjoint and positive with lowest eigenvalue given by

$$
\lambda_{0}=\pi^{2} / 4+\sigma^{2} .
$$

\section{Integral equation form}

If we introduce the inverse $K$ of the operator $A$ given in (2.21), subject to the boundary conditions (2.16), we find that $\varphi$ satisfies the equivalent integral equation

$$
F \varphi \equiv \varphi-K\{f(\varphi)\}=0,
$$

that is,

$$
\varphi(x)=\int_{0}^{1} k(x, t ; \sigma) f(\varphi(t)) d t
$$

where

$$
f(\varphi(t))=\left(\sigma^{2}-1\right)\{\varphi(t)+\xi(t)\}-\{\varphi(t)+\xi(t)\}^{3},
$$

and $k(x, t ; \sigma)$ is the kernel of $K$ given by

$$
k(x, t ; \sigma)= \begin{cases}\sinh \sigma t \cosh \sigma(1-x) / \sigma \cosh \sigma, & 0<t \leq x, \\ \sinh \sigma x \cosh \sigma(1-t) / \sigma \cosh \sigma, & x<t<1\end{cases}
$$

Since the kernel $k(x, t ; \sigma)$ is symmetric in the variables $x$ and $t$ and is Hilbert-Schmidt, $K$ is a bounded completely continuous operator [6]. Furthermore, since $K$ is a positive operator, it follows that the norm $\|K\|$ of $K$ defined by

$$
\|K\|=\max \langle K v, v\rangle \text { with }\|v\|=1,
$$

is equal to the largest eigenvalue of $K$, that is

$$
\begin{aligned}
\|K\| & =(\text { lowest eigenvalue of } \mathrm{A})^{-1} \\
& =\left(\pi^{2} / 4+\sigma^{2}\right)^{-1}
\end{aligned}
$$


We can now establish some useful inequalities involving $f, K f$ and $F$. For any functions $\varphi_{1}$ and $\varphi_{2}$ in the set $\mathcal{S}$ of (2.20) we show that there are parameters $\alpha, \beta$ and $\gamma$ such that

$$
\begin{aligned}
\left\|f\left(\varphi_{1}\right)-f\left(\varphi_{2}\right)\right\| & \leq \alpha\left\|\varphi_{1}-\varphi_{2}\right\|, & & \alpha>0, \\
\left\|K f\left(\varphi_{1}\right)-K f\left(\varphi_{2}\right)\right\| & \leq \beta\left\|\varphi_{1}-\varphi_{2}\right\|, & & 0<\beta<1,
\end{aligned}
$$

and

$$
\left\|F \varphi_{1}-F \varphi_{2}\right\| \geq \gamma\left\|\varphi_{1}-\varphi_{2}\right\|, \quad \gamma=1-\beta>0 .
$$

For the function $f$ in (3.3) we have, by the mean value theorem, that

$$
\begin{aligned}
\left\|f\left(\varphi_{1}\right)-f\left(\varphi_{2}\right)\right\| & =\left\|f^{\prime}(\theta)\left(\varphi_{1}-\varphi_{2}\right)\right\|, \quad \theta \in\left[\varphi_{1}, \varphi_{2}\right] \\
& =\left\|\left\{\sigma^{2}-1-3(\theta(t)+\xi(t))^{2}\right\}\left(\varphi_{1}-\varphi_{2}\right)\right\| \\
& \leq \max _{0 \leq t \leq 1}\left|\sigma^{2}-1-3(\theta(t)+\xi(t))^{2}\right|\left\|\varphi_{1}-\varphi_{2}\right\| .
\end{aligned}
$$

Thus (3.7) holds with

$$
\alpha=\max _{0 \leq t \leq 1}\left|\sigma^{2}-1-3(\theta(t)+\xi(t))^{2}\right| .
$$

Now choose $\sigma^{2}$ to minimize the value of $\alpha$ in (3.11). This is done by using (2.20) and taking

$$
\alpha=\sigma^{2}-1=3 u_{0}^{2}-\left(\sigma^{2}-1\right)
$$

which gives

$$
\sigma^{2}=3 u_{0}^{2} / 2+1
$$

and

$$
\alpha=3 u_{0}^{2} / 2 \text {. }
$$

Here we recall that $u_{0}$ is the prescribed end value in (2.2). For the operator $K f$ of (3.1) and (3.2) we have

$$
\begin{aligned}
\left\|K f\left(\varphi_{1}\right)-K f\left(\varphi_{2}\right)\right\| & \leq\|K\|\left\|f\left(\varphi_{1}\right)-f\left(\varphi_{2}\right)\right\| \\
& \leq \alpha\|K\|\left\|\varphi_{1}-\varphi_{2}\right\|
\end{aligned}
$$

by (3.7). Thus the inequality in (3.8) holds with

$$
\beta=\alpha\|K\|,
$$

and on using (3.6), (3.13) and (3.14) we see that

$$
\beta=\frac{u_{0}^{2}}{2}\left(\frac{\pi^{2}}{4}+\frac{3 u_{0}^{2}}{2}+1\right)^{-1},
$$


which satisfies

$$
0<\beta<1 .
$$

In this case the operator $K f$ of (3.1) and (3.2) is a contraction mapping on the set $\mathcal{S}$ which ensures the uniqueness of the solution $\varphi$ of (3.1).

To establish (3.9) for the operator $F$ defined by (3.1) we observe that

$$
\begin{aligned}
\left\|F \varphi_{1}-F \varphi_{2}\right\| & =\left\|\varphi_{1}-\varphi_{2}-\left\{K f\left(\varphi_{1}\right)-K f\left(\varphi_{2}\right)\right\}\right\| \\
& \geq\left\|\varphi_{1}-\varphi_{2}\right\|-\left\|K f\left(\varphi_{1}\right)-K f\left(\varphi_{2}\right)\right\| \\
& \geq\left\|\varphi_{1}-\varphi_{2}\right\|-\beta\left\|\varphi_{1}-\varphi_{2}\right\| \quad \text { by (3.8) } \\
& =\gamma\left\|\varphi_{1}-\varphi_{2}\right\|,
\end{aligned}
$$

where

$$
\gamma=1-\beta>0
$$

This proves (3.9).

Finally we note that if the parameter $\sigma$ is not introduced in (2.5), (2.7) and (2.8), the analysis leads to the parameter values

$$
\alpha=1+3 u_{0}^{2}, \quad \beta=\left(1+3 u_{0}^{2}\right) \frac{4}{\pi^{2}}, \quad \gamma=1-\beta,
$$

and since for $K f$ to be a contraction we require $0<\beta<1$, it follows that the values of $u_{0}$ that can be admitted are restricted to lie in the region $u_{0}{ }^{2}<\left(\pi^{2}-4\right) / 12$. Since we wish to consider values in the region $u_{0} \geq 1$ we have developed the formulation based on (2.5) which places no restriction on the end value $u_{0}$.

\section{Pointwise bounds for the solution}

We now derive pointwise bounds for the solution $\varphi(x)$ of the problem in (2.15) and (2.16), which in turn will provide bounds for the solution $u(x)$ of the original problem in (2.1) and (2.2) with (2.3). Bounds for the derivatives $\varphi^{\prime}(x)$ and $u^{\prime}(x)$ will also be derived.

By the formulation of Section 3, the function $\varphi(x)$ satisfies the integral equation

$$
\begin{aligned}
\varphi(x) & =\int_{0}^{1} k(x, t ; \sigma) f(\varphi(t)) d t \\
& =\langle k, f(\varphi)\rangle,
\end{aligned}
$$

where $k=k(x, t ; \sigma)$ and $f(\varphi)=f(\varphi(t))$ are given in (3.3) and (3.4), and the inner product is defined by (2.18) with integration over the variable $t$. Now consider the difference

$$
\Delta=\langle g, f(\varphi)\rangle-\langle g, f(\Phi)\rangle
$$


where $g$ denotes an arbitrary function in $\mathcal{L}_{2}$ and $\Phi$ is any function in $\mathcal{S}$. Then

$$
\begin{array}{rlrl}
|\Delta| & =|\langle g, f(\varphi)-f(\Phi)\rangle| & \\
& \leq\|g\|\|f(\varphi)-f(\Phi)\| & \\
& \leq\|g\| \alpha\|\varphi-\Phi\| & & \text { by (3.7) } \\
& \leq\|g\| \frac{\alpha}{\gamma}\|F \varphi-F \Phi\| & & \text { by (3.9) } \\
& =\|g\| \frac{\alpha}{\gamma}\|F \Phi\|=C(\Phi) & & \text { say, }
\end{array}
$$

using the fact that $F \varphi=0$. From (4.3) we therefore have upper and lower bounds of the form

$$
\langle g, f(\Phi)\rangle-C(\Phi) \leq\langle g, f(\varphi)\rangle \leq\langle g, f(\Phi)\rangle+C(\Phi) .
$$

Two particular choices of the function $g$ are of interest here. These are

$$
\begin{aligned}
\text { (i) } & g=k(x, t ; \sigma), \\
\text { (ii) } & g=\frac{\partial}{\partial x} k(x, t ; \sigma) .
\end{aligned}
$$

In these cases, (4.4) provides pointwise bounds for $\varphi(x)$ and its derivative $\varphi^{\prime}(x)$ respectively. These bounds, in conjunction with (2.9), then enable us to obtain pointwise bounds for $u(x)$ and its derivative $u^{\prime}(x)$, giving the solution of the original problem in (2.1) to (2.3). These bounds in (4.4) are subject to the conditions
(i) $\Phi$ belongs to $\mathcal{S}$,
(ii) $f$ satisfies (3.7),
(iii) $\quad K f$ is a contraction operator on $\mathcal{S}$.

More elaborate bounds for equations of the form (4.1) have been derived by Robinson and Yuen [5], but the bounds in (4.4) are sufficient for our purposes here.

\section{Calculations}

We now use these results to calculate pointwise bounds for the function $\varphi(x)$ and its derivative $\varphi^{\prime}(x)$, and hence for $u(x)$ and $u^{\prime}(x)$. The particular values of $u_{0}$ which will be considered here are given in Table 1 together with the associated values of $\sigma^{2}$, $\alpha, \beta$ and $\gamma$.

We start the calculations by choosing the simple trial function

$$
\Phi_{2}=c_{2} p_{2}(x), \quad p_{2}(x)=x^{2}-2 x,
$$




\begin{tabular}{|cccc|}
\hline$u_{0}$ & $\sigma^{2}$ & $\alpha$ & $\beta=1-\gamma$ \\
\hline 1 & 2.5 & 1.5 & $6 /\left(\pi^{2}+10\right)$ \\
2 & 7 & 6 & $24 /\left(\pi^{2}+28\right)$ \\
3 & 14.5 & 13.5 & $54 /\left(\pi^{2}+58\right)$ \\
\hline
\end{tabular}

TABLE 1. Values of $u_{0}$ and the associated parameters.

where the coefficients of the quadratic polynomial have been chosen so that $\Phi_{2}$ satisfies the boundary conditions

$$
\Phi(0)=0, \quad \Phi^{\prime}(1)=0 .
$$

The optimal value of the parameter $c_{2}$ is found by minimizing $\left\|F \Phi_{2}\right\|$ subject to the constraint $-u_{0}<c_{2}<0$ in order to ensure that $\Phi_{2} \in \mathcal{S}$. This in turn minimizes the correcting functional $C$. By optimizing in this way we are choosing $\Phi_{2}$ as close as possible to $\varphi$, using the fact that $\|F \varphi\|=0$. The constants $\sigma^{2}, \alpha, \beta$ and $\gamma$ are given the values indicated by Table 1 . As an illustration, for the particular case $u_{0}=1$, it is found that a minimum $\left\|F \Phi_{2}\right\|=0.004062$ is reached when $c_{2}=-0.16983$ and the resulting pointwise bounds for $u(x)$ are given in Table 2. The next step is to improve these bounds progressively by choosing polynomials of higher order for our trial function $\Phi$. Thus, let

$$
\Phi=\Phi_{3}=c_{3} p_{3}(x), \quad p_{3}(x)=a_{3} x^{3}+a_{2} x^{2}+a_{1} x+a_{0} .
$$

For this trial function to satisfy the boundary conditions (5.2) we require

$$
a_{0}=0, \quad 3 a_{3}+2 a_{2}+a_{1}=0 .
$$

Two further conditions are needed to determine the coefficients $a_{3}, a_{2}$ and $a_{1}$. From the results obtained using the quadratic trial function $\Phi_{2}$, we have an approximation for $\varphi$ given by

$$
\hat{\varphi}_{2}(x)=\left\langle k(x, t ; \sigma), f\left(\Phi_{2}\right)\right\rangle \approx \varphi .
$$

This information can be used to help choose coefficients for the next trial function $\varphi_{3}$. Thus, suppose we insist that

$$
p_{3}\left(\frac{1}{3}\right)=\hat{\varphi}_{2}\left(\frac{1}{3}\right), \quad p_{3}\left(\frac{2}{3}\right)=\hat{\varphi}_{2}\left(\frac{2}{3}\right) .
$$

Then these conditions together with (5.4) fully determine the coefficients in $\Phi_{3}$. As before, the parameter $c_{3}$ is chosen to minimize $\left\|F \Phi_{3}\right\|$ subject to the constraint $\Phi_{3} \in \mathcal{S}$. 


\begin{tabular}{|cccc|}
\hline$x$ & Lower bound & Upper bound & $C$ \\
\hline 0.2 & 0.820993 & 0.822780 & 0.000894 \\
0.4 & 0.698145 & 0.701225 & 0.001540 \\
0.6 & 0.617630 & 0.621684 & 0.002027 \\
0.8 & 0.571847 & 0.576615 & 0.002384 \\
1 & 0.556950 & 0.562041 & 0.002546 \\
\hline
\end{tabular}

TABLE 2. Pointwise bounds for $u(x)$ based on (5.1), with $u_{0}=1$.

This process can be continued to generate further trial functions $\Phi_{4}, \Phi_{5}, \ldots, \Phi_{n}, \ldots$ of increasing degrees $\mathbf{n}$. Let

$$
\Phi_{n}=c_{n} p_{n}(x), \quad p_{n}(x)=a_{n} x^{n}+a_{n-1} x^{n-1}+\cdots+a_{1} x+a_{0} .
$$

Then to satisfy (5.2) we require that

$$
a_{0}=0, \quad n a_{n}+(n-1) a_{n-1}+\cdots+a_{1}=0,
$$

which together with the $(n-1)$ conditions

$$
p_{n}(x)=\hat{\varphi}_{n-1}(x)=\left\langle k(x, t ; \sigma), f\left(\Phi_{n-1}\right)\right\rangle, \quad x=\frac{1}{n}, \frac{2}{n}, \ldots, \frac{n-1}{n},
$$

fully determine the coefficients $a_{i}$ in $p_{n}(x)$. Finally, the parameter $c_{n}$ is chosen to minimize $\left\|F \Phi_{n}\right\|$ subject to the constraint $\Phi_{n} \in \mathcal{S}$. This procedure has been continued as far as a sixth order trial function

$$
\Phi_{6}=c_{6}\left(a_{6} x^{6}+a_{5} x^{5}+\cdots+a_{1} x\right)
$$

where it is found in the case $u_{0}=1$ for example that

$$
\begin{array}{lll}
a_{1}=0.386264, & a_{2}=-0.258541, & a_{3}=-0.054139 \\
a_{4}=0.192167, & a_{5}=-0.132968, & a_{6}=1.031568
\end{array}
$$

with $c_{6}=1.00001$ and $\left\|F \Phi_{6}\right\|=0.0000053$. Pointwise bounds for $u^{\prime}(x)$ can be obtained by applying the bounds (4.4) with $g=\frac{\partial}{\partial x} k(x, t ; \sigma)$. The optimal sixth order trial function has already been found in the calculations above. The resulting pointwise bounds for both $u(x)$ and $u^{\prime}(x)$ for the cases $u_{0}=1,2,3$ are contained in Table 3. 


\begin{tabular}{|c|c|c|c|c|}
\hline & & Lower bound & Upper bound & $C$ \\
\hline \multicolumn{5}{|l|}{$u_{0}=1$} \\
\hline & $u(0.2)$ & 0.82175370 & 0.82175604 & 0.00000117 \\
\hline & $u(0.4)$ & 0.69950579 & 0.69950982 & 0.00000202 \\
\hline & $u(0.6)$ & 0.61941840 & 0.61942370 & 0.00000265 \\
\hline & $u(0.8)$ & 0.57391112 & 0.57391737 & 0.00000312 \\
\hline & $u(1)$ & 0.55913630 & 0.55914297 & 0.00000333 \\
\hline & $u^{\prime}(0)$ & -1.06700936 & -1.06699549 & 0.00000694 \\
\hline & $u^{\prime}(0.2)$ & -0.73605930 & -0.73604808 & 0.00000561 \\
\hline & $u^{\prime}(0.4)$ & -0.49751450 & -0.49750438 & 0.00000506 \\
\hline & $u^{\prime}(0.6)$ & -0.30948875 & -0.30947925 & 0.00000475 \\
\hline & $u^{\prime}(0.8)$ & -0.14871269 & -0.14870454 & 0.00000408 \\
\hline \multicolumn{5}{|l|}{$u_{0}=2$} \\
\hline & $u(0.2)$ & 1.49489235 & 1.49511356 & 0.00011061 \\
\hline & $u(0.4)$ & 1.19316988 & 1.19351110 & 0.00017061 \\
\hline & $u(0.6)$ & 1.01063135 & 1.01105410 & 0.00021137 \\
\hline & $u(0.8)$ & 0.91136837 & 0.91186457 & 0.00024810 \\
\hline & $u(1)$ & 0.87975165 & 0.88029492 & 0.00027164 \\
\hline & $u^{\prime}(0)$ & -3.30624316 & -3.30472705 & 0.00075806 \\
\hline & $u^{\prime}(0.2)$ & -1.91323035 & -1.91214030 & 0.00054503 \\
\hline & $u^{\prime}(0.4)$ & -1.16829535 & -1.16724473 & 0.00052531 \\
\hline & $u^{\prime}(0.6)$ & -0.68575207 & -0.68465900 & 0.00054653 \\
\hline & $u^{\prime}(0.8)$ & -0.31997451 & -0.31892076 & 0.00052687 \\
\hline \multicolumn{5}{|l|}{$u_{0}=3$} \\
\hline & $u(0.2)$ & 2.03766526 & 2.04083140 & 0.00158307 \\
\hline & $u(0.4)$ & 1.53785583 & 1.54218469 & 0.00216443 \\
\hline & $u(0.6)$ & 1.25643795 & 1.26139943 & 0.00248074 \\
\hline & $u(0.8)$ & 1.10878189 & 1.11455518 & 0.00288665 \\
\hline & $u(1)$ & 1.06218438 & 1.06876886 & 0.00329224 \\
\hline & $u^{\prime}(0)$ & -6.92155555 & -6.89629376 & 0.01263089 \\
\hline & $u^{\prime}(0.2)$ & -3.32910770 & -3.31217134 & 0.00846818 \\
\hline & $u^{\prime}(0.4)$ & -1.85365940 & -1.83633821 & 0.00866060 \\
\hline & $u^{\prime}(0.6)$ & -1.03882214 & -1.02043372 & 0.00919421 \\
\hline & $u^{\prime}(0.8)$ & -0.47782374 & -0.45908028 & 0.00937173 \\
\hline
\end{tabular}

TABLE 3. Pointwise bounds using a sixth order trial function. 


\section{Concluding remarks}

From Table 3 it can be seen that with simple polynomial functions the bounds (4.4) lead to close pointwise bounds for the solution. As $u_{0}$ increases these pointwise bounds grow further apart because the constant $\alpha$ increases, despite the fact that the parameter $\sigma^{2}$ has been chosen to minimize the value of $\alpha$. Nevertheless, the results obtained from (4.4) are very satisfactory considering the simple nature of the calculations. More sophisticated second order bounds can be used to improve these results further and work on these lines will be reported later.

For the particular case $u_{0}=1$, the results in Table 3 can be compared with those obtained by previous work $[1,2]$. The values of $u(1)$ and $u^{\prime}(0)$ are of special interest and from Table 3 we have

$$
\begin{aligned}
0.55913630 & \leq u(1) \leq 0.55914297 \\
-1.06700936 & \leq u^{\prime}(0) \leq-1.06699549 .
\end{aligned}
$$

The value of $u(1)$ is correct to five decimal places whereas the value of $u^{\prime}(0)$ is correct to four decimal places. These compare with the bounds obtained in [2]

$$
\begin{aligned}
0.556 & \leq u(1) \leq 0.563 \\
-1.077 & \leq u^{\prime}(0) \leq-1.049
\end{aligned}
$$

which are consistent with, but much less accurate than, the bounds resulting from the present work. The variational estimates derived in [1] are

$$
u(1) \approx 0.560, \quad u^{\prime}(0) \approx-1.022,
$$

and it can now be seen that there is quite a substantial error in the variational approximation to $u^{\prime}(0)$.

Since the procedure used here is relatively straightforward to implement, provided a suitable integral equation can be formulated, our work shows that for problems of this type the approach via bounds for $\langle k, f(\varphi)\rangle$ is an extremely effective way to find the solution.

The test case described here, with $m(u)$ given by (2.3), was chosen primarily to provide a straightforward illustration of the method. In his review of the paper, one of the referees has drawn attention to the fact that for this case the solution can be expressed in terms of a Jacobian elliptic function. Numerical work based on this provides an independent check on the results in Table 3.

Preliminary studies of other boundary value problems, in which $m(u)=1 / u^{2}$ and $m(u)=\exp (-u)$, show that the method can be applied effectively and efficiently in cases where $m(u)$ is not of simple polynomial form. 


\section{Acknowledgements}

We are indebted to the UK Science and Engineering Research Council for the award of a Research Studentship to Janet Clegg.

\section{References}

[1] N. Anderson and A. M. Arthurs, "Complementary variational principles for the steady-state finite cable model of nerve membranes", Bull. Math. Biol. 40 (1978) 735-742.

[2] A. M. Arthurs and W. M. Arthurs, "Pointwise bounds for the solution of a nonlinear problem in cell membrane theory", Bull. Math. Biol. 45 (1983) 155-168.

[3] J. J. B. Jack, D. Noble and R. W. Tsien, Electric current flow in excitable cells (Clarendon Press, Oxford, 1975).

[4] J. M. Kootsey, "The steady-state finite cable: numerical method for non-linear membrane", J. Theor. Biol. 64 (1977) 413-420.

[5] P. D. Robinson and P. K. Yuen, "Bivariational methods for Hammerstein integral equations", $I M A$ J. App. Math. 39 (1987) 177-188.

[6] I. Stakgold, Boundary value problems of mathematical physics, Volume 1 (The Macmillan Co., New York, 1967). 